\title{
Dynamical susceptibilities in a strong coupling approach
}

\author{
Andrij M. Shvaika ${ }^{a}$ \\ ${ }^{a}$ Institute for Condensed Matter Physics Nat. Acad. Sci. Ukr., 1 Svientsitskii Str., Lviv UA-79011, \\ Ukraine
}

A general scheme to calculate dynamical susceptibilities of strongly correlated electron systems within the dynamical mean field theory is developed. The approach is based on an expansion over electron hopping around the atomic limit (within the diagrammatic technique for site operators: projection and Hubbard ones) in infinite dimensions. As an example, the Falicov-Kimball and simplified pseudospin-electron models are considered and analytical expressions for the dynamical susceptibilities are obtained.

In the last decade the main achievements in the theory of the strongly correlated electron systems are connected with the development of the Dynamical Mean Field Theory (DMFT) which is exact in the $d=\infty$ limit [1]. It was shown by Metzner and Vollhardt [2,3] that in the $d=\infty$ limit self-energies are single-site quantities (do not depend on wave vector) which leads to a significant simplification. The same is true for the four-vertices in the Bethe-Salpeter equation for susceptibilities (see [1]).

The aim of this article is to develop a general scheme to calculate dynamical susceptibilities within a diagrammatic technique for site operators (projection or Hubbard ones) for strongly correlated electron systems described by the general statistical operator

$\hat{\rho}=e^{-\beta \hat{H}_{0}} \times$

$T \exp \left\{-\int_{0}^{\beta} d \tau \int_{0}^{\beta} d \tau^{\prime} \sum_{i j \sigma} t_{i j}^{\sigma}\left(\tau-\tau^{\prime}\right) a_{i \sigma}^{\dagger}(\tau) a_{j \sigma}\left(\tau^{\prime}\right)\right\}$,

where $\hat{H}_{0}=\sum_{i} \hat{H}_{i}$ is a sum of the single-site contributions. Our approach is based on an expansion over electron hopping around the atomic limit [3] (see also [4]) instead of an expansion in the local interaction [1].

In the $d=\infty$ limit, the lattice problem with $t_{i j}^{\sigma}\left(\tau-\tau^{\prime}\right)=\delta\left(\tau-\tau^{\prime}\right) t_{i j} / \sqrt{d}$, is mapped onto an effective atomic problem with a dynamical mean field $t_{i j}^{\sigma}\left(\tau-\tau^{\prime}\right)=\delta_{i j} J_{\sigma}\left(\tau-\tau^{\prime}\right)$. Single-electron Green's functions for the lattice $G_{\sigma}\left(\omega_{\nu}, \boldsymbol{k}\right)=$
$\left[\Xi_{\sigma}^{-1}\left(\omega_{\nu}\right)-t_{\boldsymbol{k}}\right]^{-1}$ and the effective atomic problem $G_{\sigma}^{(a)}\left(\omega_{\nu}\right)=\left[\Xi_{\sigma}^{-1}\left(\omega_{\nu}\right)-J_{\sigma}\left(\omega_{\nu}\right)\right]^{-1}$ are determined by the same single-site irreducible parts $\Xi_{\sigma}\left(\omega_{\nu}\right)$ [3] and a closed set of equations for $\Xi_{\sigma}\left(\omega_{\nu}\right)$ and $J_{\sigma}\left(\omega_{\nu}\right)$ can be written 11 . Here, irreducible parts are contributions to the single-site Green's function which cannot be divided into parts by cutting one hopping line.

In a similar way, the expansion for correlation functions built on operators $\hat{A}$ and $\hat{B}$ in terms of the hopping is

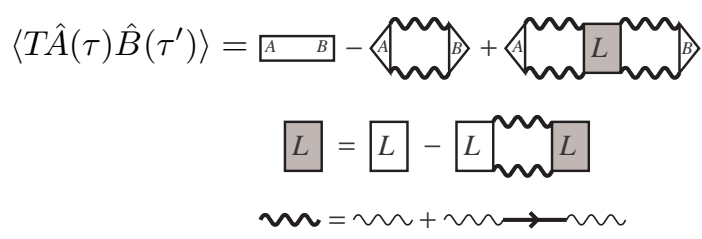

where thin wavy lines denote electron hopping integrals $t_{i j}^{\sigma}\left(\tau-\tau^{\prime}\right)$ and arrows denote singleparticle Green's functions. In eq. (2), $\square,\langle$ and $L$ are single-site quantities, which are the same for the lattice and the effective atomic problems and are generalized many-particle Green's functions [4] which will be calculated within DMFT.

To do this, we calculate a two-particle Green's function for the effective atomic problem

$\left\langle T a_{1}^{\dagger} a_{2} a_{3}^{\dagger} a_{4}\right\rangle_{(a)} \equiv{ }_{2}^{1} \Upsilon_{3}^{4}$,

which, on the other hand, can be written in the following way:

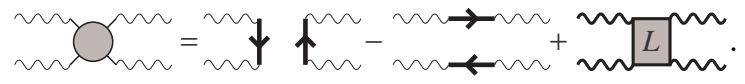


Combining this equation with the equation for the four-vertex of the atomic problem allows us to calculate the single-site four-vertex $L$. Similarly, we can calculate other single-site vertices $\square$ and $\$ and, finally, determine the correlation functions (2) for the lattice.

As an example, we consider a binary alloy model

$H_{i}=\frac{g}{2} P_{i}^{+} n_{i}-\frac{g}{2} P_{i}^{-} n_{i}-\frac{h}{2}\left(P_{i}^{+}-P_{i}^{-}\right)-\mu n_{i}$,

where $P_{i}^{ \pm}=\frac{1}{2} \pm S_{i}^{z}$ for the $U=0$ pseudospinelectron (PE) model, $P_{i}^{+}=c_{i}, P_{i}^{-}=1-c_{i}$ for a binary alloy, and $a_{i \sigma} \rightarrow d_{i}, n_{i}=d_{i}^{\dagger} d_{i}, P_{i}^{+}=$ $f_{i}^{\dagger} f_{i}, P_{i}^{-}=1-f_{i}^{\dagger} f_{i}$ for the Falicov-Kimball (FK) model [5]. The single-particle Green's function for the effective atomic problem is a coherent sum of the Green's functions for subspaces $S_{i}^{z}= \pm \frac{1}{2}$ and is equal to [6,7]

$$
\begin{aligned}
G_{\sigma}^{(a)}\left(\omega_{\nu}\right) & =\frac{\left\langle P^{+}\right\rangle}{i \omega_{\nu}+\mu-J_{\sigma}\left(\omega_{\nu}\right)-\frac{g}{2}} \\
& +\frac{\left\langle P^{-}\right\rangle}{i \omega_{\nu}+\mu-J_{\sigma}\left(\omega_{\nu}\right)+\frac{g}{2}}
\end{aligned}
$$

which allows us to find solutions for $\Xi_{\sigma}\left(\omega_{\nu}\right)$ and $J_{\sigma}\left(\omega_{\nu}\right)$.

For the model (3) all many-particle Green's functions for the effective atomic problem can be obtained in an analytical form which allows us to calculate dynamical susceptibilities: pseudospin

$\chi^{S^{z} S^{z}}\left(\omega_{m}, \boldsymbol{q}\right)=\frac{\delta\left(\omega_{m}\right) \Delta_{S^{z}}^{2}}{T-\Theta(T, \boldsymbol{q})}$,

charge

$\chi^{n n}\left(\omega_{m}, \boldsymbol{q}\right)=\frac{\delta\left(\omega_{m}\right) \Delta_{n}^{2}}{T-\Theta(T, \boldsymbol{q})}+K^{n n}\left(\omega_{m}, \boldsymbol{q}\right)$

and mixed

$\chi^{n S^{z}}\left(\omega_{m}, \boldsymbol{q}\right)=\chi^{S^{z} n}\left(\omega_{m}, \boldsymbol{q}\right)=\frac{\delta\left(\omega_{m}\right) \Delta_{n} \Delta_{S z}}{T-\Theta(T, \boldsymbol{q})}$,

where

$\Theta(T, \boldsymbol{q})=\frac{1}{\beta} \sum_{\nu \sigma} \frac{\Lambda_{\sigma \nu}^{2}\left(\chi_{\sigma \nu 0}(\boldsymbol{q})-\tilde{\chi}_{\sigma \nu}\right)}{\tilde{\chi}_{\sigma \nu 0}^{2}+\Lambda_{\sigma \nu}^{2}\left(\chi_{\sigma \nu 0}(\boldsymbol{q})-\tilde{\chi}_{\sigma \nu 0}\right)}$,

$$
\begin{aligned}
& K^{n n}\left(\omega_{m}, \boldsymbol{q}\right)= \\
& \frac{1}{\beta} \sum_{\nu \sigma} \frac{\tilde{\chi}_{\sigma \nu m}\left(\tilde{\chi}_{\sigma \nu m}-\Lambda_{\sigma \nu} \Lambda_{\sigma \nu+m}\right) \chi_{\sigma \nu m}(\boldsymbol{q})}{\tilde{\chi}_{\sigma \nu m}^{2}+\Lambda_{\sigma \nu} \Lambda_{\sigma \nu+m}\left(\chi_{\sigma \nu m}(\boldsymbol{q})-\tilde{\chi}_{\sigma \nu m}\right)}, \\
& \Lambda_{\sigma \nu}=\frac{g \sqrt{\left\langle P^{+}\right\rangle\left\langle P^{-}\right\rangle}}{\left(i \omega_{\nu}+\mu-J_{\sigma}\left(\omega_{\nu}\right)\right)^{2}-\frac{g^{2}}{4}}, \\
& \Delta_{n}=\frac{1}{\beta} \sum_{\nu \sigma} \frac{\Lambda_{\sigma \nu} \tilde{\chi}_{\sigma \nu 0} \chi_{\sigma \nu 0}(\boldsymbol{q})}{\tilde{\chi}_{\sigma \nu 0}^{2}+\Lambda_{\sigma \nu}^{2}\left(\chi_{\sigma \nu 0}(\boldsymbol{q})-\tilde{\chi}_{\sigma \nu 0}\right)}, \\
& \Delta_{S z}=\sqrt{\left\langle P^{+}\right\rangle\left\langle P^{-}\right\rangle}, \\
& \chi_{\sigma \nu m}(\boldsymbol{q})=-\frac{1}{N} \sum_{\boldsymbol{k}} G_{\sigma}\left(\omega_{\nu}, \boldsymbol{k}\right) G_{\sigma}\left(\omega_{\nu+m}, \boldsymbol{k}+\boldsymbol{q}\right), \\
& \tilde{\chi}_{\sigma \nu m}=-G_{\sigma}\left(\omega_{\nu}\right) G_{\sigma}\left(\omega_{\nu+m}\right) .
\end{aligned}
$$

The expression for $\Theta(T, \boldsymbol{q})$ coincides with the one obtained by Freericks $[8]$ and it is known that the ground state of the model (3), for a fixed average value of the pseudospin, is not uniform and shows either commensurate order, incommensurate order, or phase separation [8]. On the other hand, in the case of a fixed value of the field $h$ the possibility of a uniform first-order phase transition (bistability) appears [7,9].

\section{REFERENCES}

1. A. Georges, G. Kotliar, W. Krauth, M.J. Rosenberg, Rev. Mod. Phys. 68 (1996) 13.

2. W. Metzner, D. Vollhardt, Phys. Rev. Lett. 62 (1989) 324.

3. W. Metzner, Phys. Rev. B 43 (1991) 8549.

4. A.M. Shvaika, cond-mat/9911255.

5. L.M. Falicov, J.C. Kimball, Phys. Rev. Lett. 22 (1969) 997.

6. U. Brandt, C. Mielsch, Z. Phys. B 75 (1989) 365; 79 (1990) 295; 82 (1991) 37.

7. I.V. Stasyuk, A.M. Shvaika, J. Phys. Studies 3 (1999) 177.

8. J.K. Freericks, Phys. Rev. B 47 (1993) 9263.

9. W. Chung, J.K. Freericks, Phys. Rev. B 57 (1998) 11955. 